\title{
Research on Anti Impact Simulation of Sensor Electronic Equipment
}

\author{
Kai Li ${ }^{1}$, and Yan $\operatorname{Han}^{1, b}$ \\ ${ }^{1}$ Shanxi Key Laboratory of Signal Capturing and Processing, National Key Laboratory for Electronic \\ Measurement Technology, North University of China, Taiyuan, 030051, China \\ alikai@nuc.edu.cn, bhanyan@nuc.edu.cn
}

\begin{abstract}
Keywords: Sensor electronic equipment; Vibration isolation; Vibration; Shock; Finite element simulation.

Abstract. This paper analyses the mechanism of impact vibration on electronic equipment of the sensor and the vibration isolation, as well as buffering mechanism of cushioning materials. And for the impact environment of electronic equipment of the sensor, the design of the structure against the impact of high overload is made. The vibration isolator of the sensor is developed by using both of potting material and the rubber - foam aluminum overlap method. The shock wave is attenuated by a multi - layer dielectric absorption technique, and the shock resistance of the sensor system is improved. ANSYS software obtains the maximal stress, maximum displacement, stress time curve, and displacement time curve of the system for transient impact analysis of the electronic sensor equipment. The simulation results validate the rationality of the design method against high overload impact.
\end{abstract}

\section{Introduction}

The sensor electronics are subject to the interference of mechanical forces, such as vibration, impact and centrifugal force being used in the course. Among them, vibration and impact are main factors that cause a failure of the electronic equipment of the sensor. Results of related researches show that the failure rate of the electronic system caused by vibration and impact is as high as $50 \% \sim 60 \%[1-2]$. The electronic equipment of the sensor is subject to the vibration impact of $12 \mathrm{~g}$ or less in a daily storage process[3], and the instantaneous, high-intensity impact of up to $10,000 \mathrm{~g}$ or more when it is used. In particular circumstances, the maximum impact acceleration may be $100,000 \mathrm{~g}$ or more[4]. Conventional sensor electronics generally can only withstand an overload acceleration of tens of $g$, and it is necessary to take certain protection measures for the electronic sensor equipment to ensure the normal operation of the system in the applications, such as vibration measurement, impact measurement and acceleration measurement[5-6]. Therefore, the technology of the sensor electronic equipment against high overload impact is the core technology of intelligence and information of the sensor system[7-8].

Currently, the impact resistance of the electronic equipment of the sensor is improved by increasing the inherent frequency of the circuit board to reduce the probability of resonance[9]. The main method to increase overload is potting, which uses the potting material with excellent dynamic performance to encapsulate the sensor so that it may be cured into a module. But the inherent frequency of the sensor system may also be improved, and the fatigue effect of the system may be reduced through the miniature design of the sensor electronics[10].

The core device of sensor electronics is PCB circuit, so this paper focuses on analyzing the impact resistance of PCB circuit and the damage mechanism of electronic sensor equipment in a vibration environment, and proposes the damage mechanism of sensor electronics in vibration and impact environment. To ensure that the electronic sensor equipment can withstand $10,000 \mathrm{~g}$ transient high overload impact, a design integrating the potting technology, vibration isolation technology, and multi-layer dielectric absorption technology is applied to improve the impact resistance of the electronic equipment of the sensor[11-13]. And a modal analysis and high-impact transient analysis 
of the sensor electronics are provided by ANSYS numerical simulation, which provides a reference method for the performance of the electronic sensor equipment against high overload impact.

\section{Impact resistant design}

\section{The damage mechanism of the impact on the electronic equipment of the sensor}

The electronic sensor equipment will produce a vibration with a frequency of $2 \mathrm{kHz}$ or less in the process of transport, and if its inherent frequency is less than $2 \mathrm{kHz}$, the probability of high amplitude resonance will become larger. If the sensor electronic equipment is subject to an external transient impact in use, the maximum overload amplitude of the gravity wave generated by the process may reach tens of thousands of $\mathrm{g}$; the high overload stress wave will make the PCB circuit board crack or even fracture, and also make the electronic element severely deformed and fail[14].

The damage ways of vibration and high impact on the electronic equipment of the sensor are as follows: the frequency of the external impact gets close to the inherent frequency of the sensor to resonant, and the vibration acceleration thus generated exceeds the overload resistance of the sensor, destroying the sensor; the external vibration or shock acceleration continues to occur, and the sensor produces fatigue effect due to long-term vibration or impact, degrading or damaging the performance of the sensor.

According to Steinberg formula[15], the following relationship between the maximum vibration amplitude and the inherent frequency of the guided electronic equipment is derived:

$$
A_{M A X}=\frac{9.8 G_{\text {out }}}{f_{n}^{2}}=\frac{9.8 G_{\text {in }}}{f_{n}^{3 / 2}}
$$

Wherein, $f_{n}$ is the inherent frequency of the PCB circuit, $G_{\text {out }}$ is the acceleration at the maximum amplitude of the PCB circuit, the unit is $\mathrm{g}$, and $G_{\text {out }}=G_{\text {in }} Q ; G_{\text {in }}$ is the acceleration of excitation load, and the unit is $\mathrm{g}$; $Q$ is the excitation transfer rate of $\mathrm{PCB}$, and $Q=f_{n}{ }^{1 / 2}$.

According to the equation (1), to reduce the maximum amplitude, it is necessary to improve the system's inherent frequency. The miniaturized structure has high rigidity and strength, so its inherent frequency is higher. Thus, a chip-type electronic component is used to reduce the mass and volume of the structure. Meanwhile, the mass of the circuit board is uniformly distributed without affecting the performance of the circuit, thus not only improving the inherent frequency but also preventing the electronic system from concentrated stress.

In order to ensure that the electronic equipment of the sensor system can work properly, according to the damage modes of vibration or impact the sensor electronic equipment is subjected to, two methods are designed to improve the vibration or impact resistance performance of the sensor electronic equipment: increase the inherent frequency and reduce the probability of resonance of the sensor by optimizing its structure; increase the strength of the sensor and improve its impact resistance by adding a vibration isolation device.

\section{Vibration isolation mechanism}

The purpose of the vibration isolation device is to absorb the kinetic energy of the sensor electronics and to attenuate the stress wave so that the kinetic energy of the electronic device itself may be absorbed by the cushioning material while reducing the magnitude of the stress wave. Figure 1 is a stress-strain curve of the cushioning material covering three stages: elastic deformation, yield platform, and material compaction[16]. The cushioning material undergoes a long yielding stage before compacting. This property determines the energy absorption properties of the cushioning material and the yield plateau of the material is relatively small. The material will not transfer a force above the platform value before being compacted. The buffer material has a viscoelastic effect and transverse inertia effect, so that the stress wave will have amplitude attenuation and wave dispersion during the propagation process. 


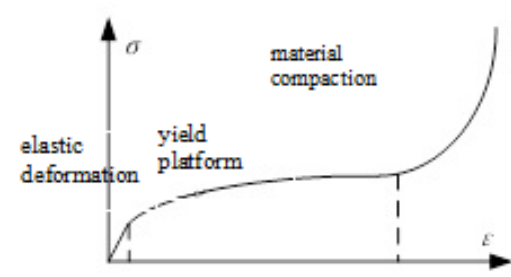

Fig.1 Stress-strain curve of potting materials

\section{Impact resistant design of the electronic sensor equipment}

In order to make the impact resistance of the electronic sensor equipment reach $10,000 \mathrm{~g}$, this study designs from the potting technology, vibration isolation technology, and multi-layer dielectric absorption technology to improve the system's impact resistance.

1) Potting technology is a non-porous packaging of the sensor to solidify it into a module and is a process of energy absorption using elastic-plastic deformation and damping effect of the cushioning material. Polyurethane material features low hardness, good elasticity, strong bond, and good electrical properties, as well as excellent insulation, insulation and corrosion resistance, so polyurethane is selected as potting material.

2) The vibration isolator is designed with vibration isolation technology so that the isolator can store the energy of impact, and the energy stored after the end of the impact may be released in the form of free attenuation. The vibration isolator can be classified into metal type, rubber type, and air type. The rubber material and foam aluminum are chosen as the vibration isolator in this structure. The rubber material features low stiffness, light weight, large damping capacity and can absorb more impact energy. Foam aluminum have a very high porosity with a non-uniform structure and a strain lagging behind the stress, and the stress and strain curve has a very long straight line segment, so it is a high damping material with high energy absorption.

3) The inevitable reflection and refraction phenomena in the spread of shock wave in the interfaces of different media lead to an energy attenuation, which is called the multi-layer dielectric absorption technology. Rubber material and foam aluminum are alternately superposed as the vibration isolator of the electronic sensor equipment in the design of vibration isolators.

\section{Numerical analysis}

\section{Basic assumptions and model building}

In this paper, ANSYS finite element software is used to simulate the impact of high overload for the structure, and the model is calculated based on the following basic assumptions: all materials are the uniform continuous media; the mass of small components is uniformly distributed to the circuit board using the total mass equivalent method due to their small size and mass; impact process is an adiabatic process.

1. Simulation model design

There are miniature parallelepipedic PABC voxels in the sensor electronics with $\mathrm{PA}=\mathrm{dx}, \mathrm{PB}=\mathrm{dy}$, $\mathrm{PC}=\mathrm{dz}$. The stress on each face is decomposed into a normal stress and two shear stresses, parallel to the three axes.

The stress state at any point within an electronic device can be represented by six stress components $\sigma_{\mathrm{x}}, \sigma_{\mathrm{y}}, \sigma_{\mathrm{z}}, \tau_{\mathrm{x}}, \tau_{\mathrm{y}}, \tau_{\mathrm{z}}, \tau_{\mathrm{xy}}, \tau_{\mathrm{yz}}$ and $\tau_{\mathrm{xz}}$. In general, the stress states at different points in the elastomer are not the same. Therefore, the above six stress components describing the stress state of the elastomer are not constants, but a function of the coordinates $\mathrm{x}, \mathrm{y}$ and $\mathrm{z}$.

Experiments show that the stress components caused by the three stresses are superposed to obtain the component of synthesized strain. Assume that the elastic body undergoes positive stress uniformly distributed on each surface. According to the generalized Hooke's law, the total of the six stress components can be represented by a column matrix $\{\sigma\}$ : 


$$
\left\{\begin{array}{c}
\varepsilon_{\mathrm{x}}=\frac{1}{\mathrm{E}}\left[\sigma_{\mathrm{x}}-\mu\left(\sigma_{\mathrm{y}}+\sigma_{\mathrm{z}}\right)\right] \\
\varepsilon_{\mathrm{y}}=\frac{1}{\mathrm{E}}\left[\sigma_{\mathrm{y}}-\mu\left(\sigma_{\mathrm{x}}+\sigma_{\mathrm{z}}\right)\right] \\
\varepsilon_{\mathrm{z}}=\frac{1}{\mathrm{E}}\left[\sigma_{\mathrm{z}}-\mu\left(\sigma_{\mathrm{x}}+\sigma_{\mathrm{y}}\right)\right] \\
\gamma_{\mathrm{xy}}=\frac{\tau_{\mathrm{xy}}}{\mathrm{G}} \\
\gamma_{\mathrm{yz}}=\frac{\tau_{\mathrm{yz}}}{\mathrm{G}} \\
\gamma_{\mathrm{zx}}=\frac{\tau_{\mathrm{zx}}}{\mathrm{G}}
\end{array}\right.
$$

Written in matrix form:

$\{\sigma\}=[\mathrm{D}]\{\varepsilon\}$

[D] is called the elastic matrix, and is completely determined by the elastic constants $\mathrm{E}$ and $\mu$

For the system at equilibrium under the action of the force, when the displacement of the rigid body coincides with the restraint condition, the total work (the algebraic sum of the work done by the forces) of all the active forces in the system is constant to zero. According to the principle of virtual work:

$\{\sigma\}^{\mathrm{T}}\{\mathrm{F}\}=\iiint\{\varepsilon\}^{\mathrm{T}}\{\sigma\} \mathrm{dxdydz}$

The relationship between the external force and the stress is represented by the imaginary displacement and the imaginary strain of the above equation. With the principle of imaginary displacement, only an integral equation is needed to solve the displacement, and it can be proved that the solution of the extreme value problem and the solution of the differential equation are consistent, and both are equivalent under the condition of satisfying the deformation coordination equation.

Displacement is solved by external force by virtual displacement instead of equilibrium differential equation, and the displacement equation of each element is simplified by partitioning grid, which is easy to set up and solve.

2. Simulation of the impact of sensor

The three-dimensional solid element Solid45 is used in the aluminum housing and the sensor electronics while super-elastic element Solid185 is used in vibration isolator and potting material. The sensor electronics and the aluminum housing material are linear elastic models, the rubber material, and the potting materials are Mooney-Rivilin models, and the aluminum foam is bilinear isotropic stiffening linear reinforced elastoplastic material. The solid model of the sensor electronics is shown in Fig 2.

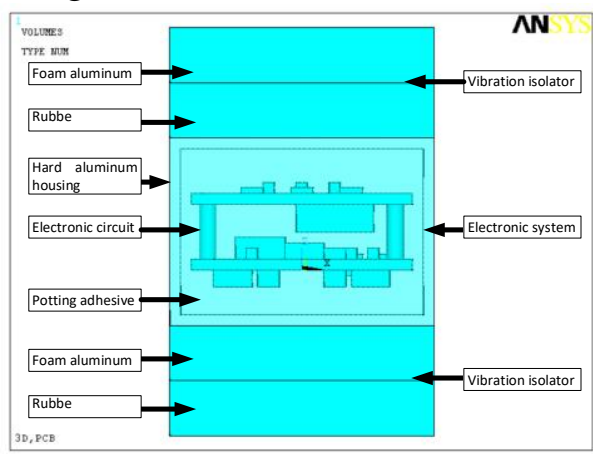

Figure 2 The overall model of the sensor electronic equipment

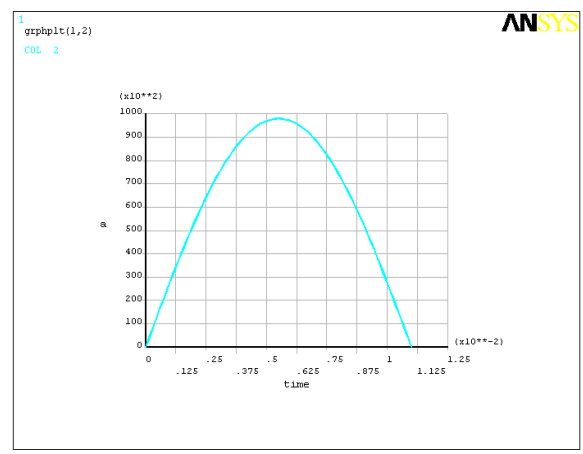

Figure 3 Acceleration load curve

\section{Impact simulation of sensor electronic equipment}

Sensor electronic equipment should withstand a transient impact load of 10,000g. According to the impact test method provided for electronic sensor equipment in the standard GJB150.18-86, the impact pulse commonly used in sensor electronics is half-sine wave pulse, and the pulse time is $11 \mathrm{~ms}$. The load curve is shown in Fig 3. 
Since ANSYS cannot directly load the transient acceleration load, the researchers usually integrate the acceleration load twice to obtain a displacement curve of the impact as a displacement load imposed on the model. This loading method has two deficiencies: (1)The displacement curve obtained by this method is the whole system displacement, while the researchers are concerned about the relative displacement of the system; (2)At the end of the actual impact load, the system is damped with damped vibration; however, the displacement continues to increase after the end of the shock in the simulation, which does not match the actual situation[17]. In order to overcome the above two shortcomings, this paper uses an equivalent inertial force load instead of an equivalent displacement load. First, the system mass matrix is extracted, and then the equivalent inertial force is obtained according to $F=m a$.

The time-history post-processor POST26 can be used to view the stress-displacement and time-dependent displacement of the model-specified nodes. Figure 4 shows the stress-time-dependent curves of the nodes at the maximum stress. Figure 5 shows the displacement versus time of the maximum displacements. It can be concluded that the maximum stress and maximum displacement of the sensor electronics occur at $6.27 \mathrm{~ms}$. Using the general postprocessor POST1, a cloud of stress and displacement of the sensor electronics at $\mathrm{t}=6.27 \mathrm{~ms}$ can be obtained. As shown in Fig. 6, the maximum stress occurs near the four screw holes and the connection between the electronic components and the PCB board, the maximum value is $68.3 \mathrm{MPa}$, and the allowable stress of the PCB board is as high as $196 \mathrm{MPa}$, so the structure of the electronic equipment will not be destroyed. As shown in Figure 7, the maximum displacement occurs on one side of the PCB board with a maximum of $0.0275 \mathrm{~mm}$. According to the Lauetal criterion[18]: When the maximum amplitude of the PCB is greater than $1.5 \mathrm{~mm}$, the surface of the component on the PCB will lead to fatigue failure of the pin while the maximum deformation of the structure is only $0.0115 \mathrm{~mm}$, in the allowable range.

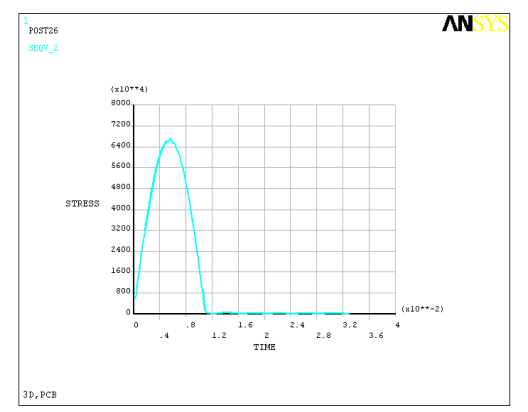

Fig.4 Curve of equivalent stress versus time

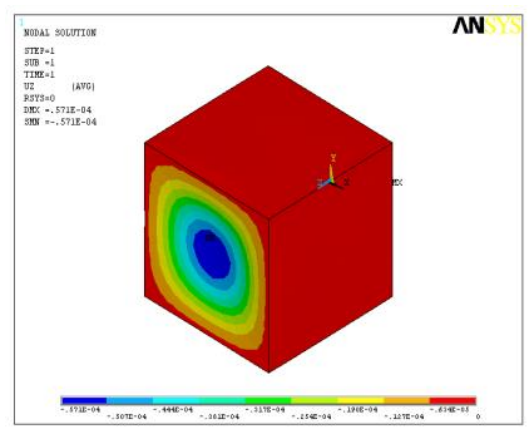

Figure 7 Stress of the overall model $(5,000 \mathrm{G})$

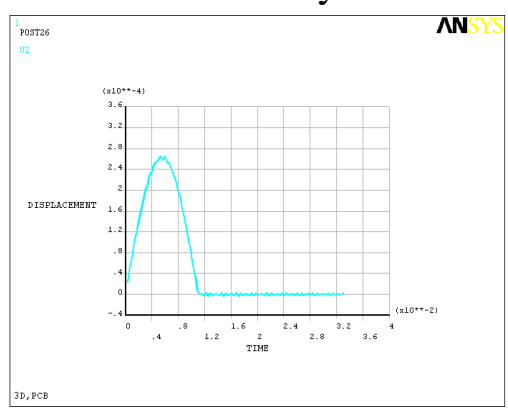

Figure 5 Curve of displacement versus time

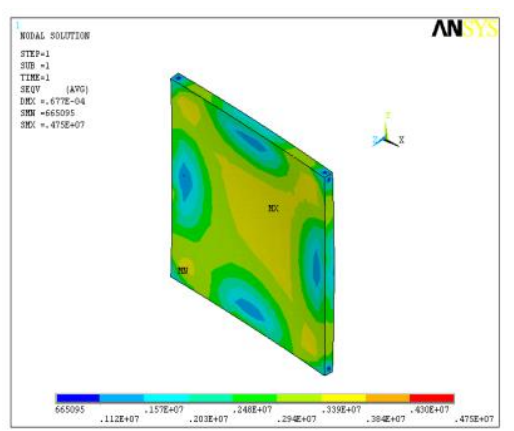

Figure 8 Stress of PCB board $(5,000 \mathrm{G})$

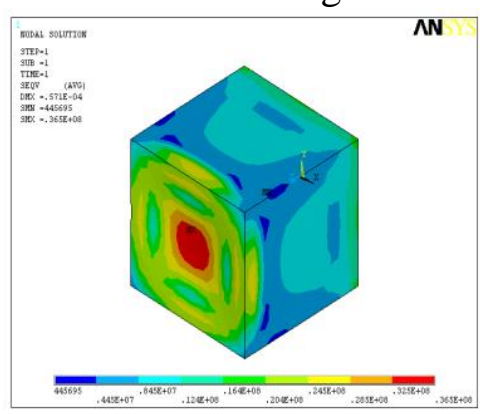

Figure 6 Displacement of the overall model $(5,000 \mathrm{G})$

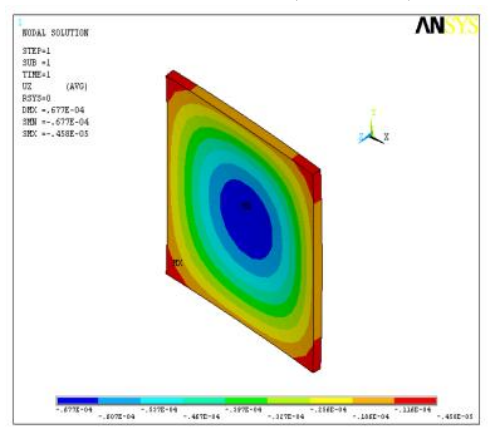

Figure 9 Displacement of PCB board $(5,000 \mathrm{G})$

Figure 6 shows the minimum stress of the overall model is $0.445 \mathrm{MPa}$ and the maximum stress is $36.5 \mathrm{MPa}$. Figure 7 shows the minimum displacement of the overall model is $0.0063 \mathrm{~mm}$ and the maximum displacement is $0.0571 \mathrm{~mm}$.

Figure 8 shows the minimum stress of PCB board is $0.665 \mathrm{MPa}$, and the maximum stress is 4.75MPa. Figure 9 shows the minimum displacement of PCB board is $0.0046 \mathrm{~mm}$, and the maximum displacement is $0.0677 \mathrm{~mm}$. 
Figure 10 shows the chip has a minimum displacement of $0.0416 \mathrm{~mm}$ and a maximum displacement of $0.0425 \mathrm{~mm}$. Figure 11 shows the chip has a minimum stress of $0.699 \mathrm{MPa}$ and a maximum stress of $60.5 \mathrm{MPa}$.

Figure 12 shows the solder has a minimum displacement of $0.0358 \mathrm{~mm}$, and a maximum displacement of $0.0381 \mathrm{~mm}$. Figure 13 shows the solder has a minimum stress of $0.237 \mathrm{MPa}$ and a maximum stress of $26.2 \mathrm{MPa}$.

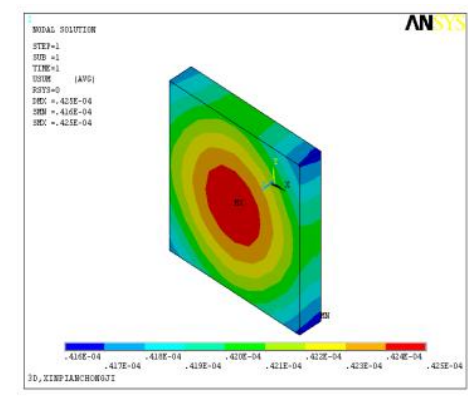

Figure 10 Chip displacement

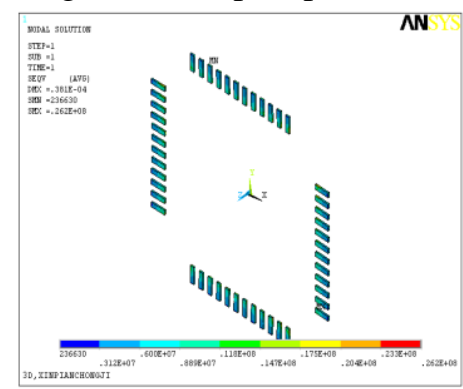

Figure 13 Solder stress

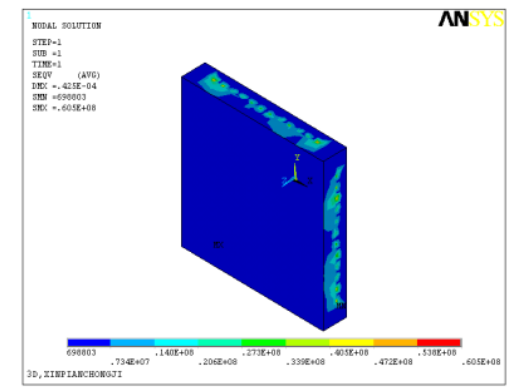

Figure 11 Chip stress

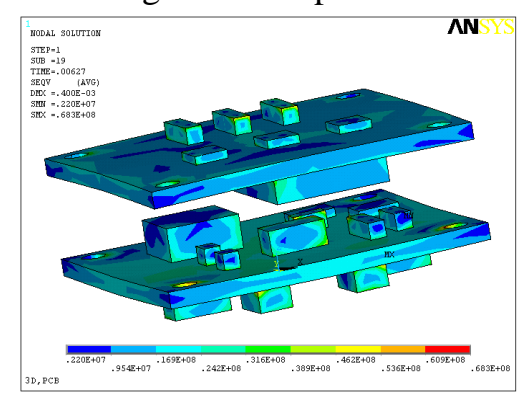

Figure 14 Equivalent stress cloud

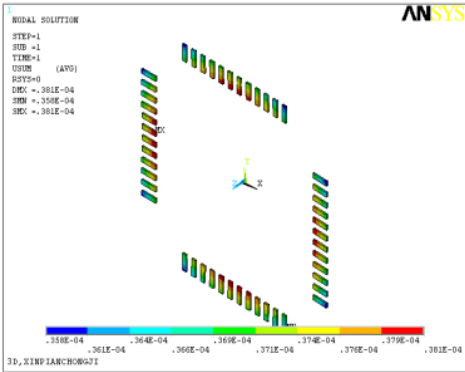

Figure 12 Solder displacement

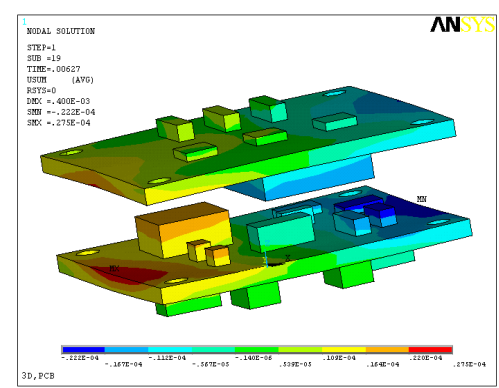

Figure 15 Displacement cloud

Figure 14 shows the equivalent stress cloud for electronic equipment at $t=6.27 \mathrm{~ms}$. Figure 14 shows the displacement cloud for electronic equipment at $t=6.27 \mathrm{~ms}$.

\section{Conclusion}

(1) The sensor system is designed with high overload impact resistance based on the impact environment of the electronic equipment and the structural characteristics of the elastomer from the three aspects of potting technology, vibration isolation technology, and multi-layer dielectric absorption technology according to the damage mode of the impact on electronic equipment.

(2) In the paper, the impact of the structure designed is analyzed by ANSYS software. The results of transient shock analysis show that the maximum stress of $58.2 \mathrm{MPa}$ appears at the screw hole position and the maximum displacement is $0.0115 \mathrm{~mm}$. The numerical results show that the system can withstand 10,000g overload impact without damage to the structure.

(3) ANSYS finite element design method replaces the traditional "design - experiment - design" cycle, which significantly improves the efficiency of the system structure design, shorten the design cycle and reduce costs. The design method of this study also applies to the impact resistant design of a variety of fuses.

\section{References}

[1] Bruce Kaufman.A Nonvolatile Solid-State Recorder for 100,000g.Environments. Sensors Magazine .Vol. 17-6(2000), P 46-51.

[2] Bruce Kaufman .This Solid-State Recorder Rides in a Bullet .Sensors Magazine .Vol. 7-3(2005), P 4-5.

[3] C. Gradolph, A. Friedberger, G. Müller, J. Wilde, Impact of high-g and high vibration environments on piezoresistive pressure sensor performance, Sensors and Actuators A: Physical, Vol. 150-1(2009), P 69-77. 
[4] Shi Yunbo, Li Ping, Zhu Zhengqiang, Liu Jun, Zhang Xiaoming. High overload ability optimization of a MEMS high-g accelerometer, Journal of Vibration and Shock, Vol. 30-7(2011), P 271-274.

[5] Qian Lizhi,Study of Projectile-loaded Equipment Against High Overload, Acta armamentarii,2007,Vol. 28-8(2007),P 1017-1020.

[6] Han Shuai,Ma Youchun,Qin Li,Wang Yuekai,Ding Ning.MEMS piezoresistive sensor based design of low-power consuming and high-overloaded testing system,Explosion and Shock Waves, Vol. 36-5(2016), P 721-727.

[7] Claudia F. Castillo-Berrio, Vicente Feliu-Batlle, Vibration-free position control for a two degrees of freedom flexible-beam sensor, Mechatronics, Vol. 27(2015), P 1-12.

[8] Satish Mohanty, Karunesh K. Gupta, Kota Solomon Raju, Vibration Feature Extraction and Analysis of Industrial Ball Mill Using MEMS Accelerometer Sensor and Synchronized Data Analysis Technique, Procedia Computer Science, Vol. 58(2015), P 217-224.

[9] A.L. Araújo, V.S. Carvalho, C.M. Mota Soares, J. Belinha, A.J.M. Ferreira, Vibration analysis of laminated soft core sandwich plates with piezoelectric sensors and actuators, Composite Structures, Vol. 151-1(2016), P 91-98.

[10] Ricardo de Medeiros, Murilo Sartorato, Dirk Vandepitte, Volnei Tita, A comparative assessment of different frequency based damage detection in unidirectional composite plates using MFC sensors, Journal of Sound and Vibration, Vol. 383-24(2016), P 171-190.

[11] Mariia Polikarpova, Pia Lindh, Chris Gerada, Marko Rilla, Ville Naumanen, Juha Pyrhönen, Thermal effects of stator potting in an axial-flux permanent magnet synchronous generator, Applied Thermal Engineering, Vol. 75-22(2015), P 421-429.

[12]P. Veeramuthuvel, K.K. Sairajan, K. Shankar, Vibration suppression of printed circuit boards using an external particle damper, Journal of Sound and Vibration, Vol. 366-31(2016), P 98-116.

[13] Jianfeng Yang, Zhenbang $\mathrm{Xu}$, Qingwen Wu, Mingchao Zhu, Shuai He, Chao Qin, Dynamic modeling and control of a 6-DOF micro-vibration simulator, Mechanism and Machine Theory, Vol. 104(2016), P 350-369.

[14]Zhaoxia Ma, Weiping Xie, Guangcai Yan, Wei He, Numerical Analysis and Tests on Vibration Reduction Effects of a New Vibration Isolation Module, Procedia Earth and Planetary Science, Vol. 5(2012), P 47-51.

[15] Kouta Kunugi, Hirohisa Kojima, Pavel M. Trivailo, Modeling of tape tether vibration and vibration sensing using smart film sensors, Acta Astronautica, Vol. 107(2015), P 97-111.

[16] Arrayago, E. Real, L. Gardner, Description of stress-strain curves for stainless steel alloys, Materials \& Design, Vol. 87(2015), P 540-552.

[17] Qinghua Meng, Zhenqing Wang, Numerical simulation of loading edge cracks by edge impact using the extended finite element method, Acta Mechanica Solida Sinica, Vol. 28-2(2015), P 156-167.

[18] Javier Toraňo, Rafael Rodríguez, Isidro Diego, José M. Rivas, María D. Casal, FEM models including randomness and its application to the blasting vibrations prediction, Computers and Geotechnics, Vol. 33-1(2006), P 15-28. 